\title{
Estimation of Seminal MDA Levels in Infertility patients
}

\author{
Dr. Shazia Masroor, Dr. J. N. Muneshwar, Dr. U. S. Zingade \\ Department of Physiology, Govt. Medical College, Aurangabad
}

\begin{abstract}
Background- Defective sperm function is the most common cause of infertility. There is growing evidence that damage to spermatozoa by Reactive Oxygen Species(ROS) play a key role in male infertility. Oxidative stress is a common pathology seen in approximately half of all infertile men. Mammalian spermatozoa are rich in poly unsaturated fatty acids (PUFA) and are very susceptible to attack by ROS, leading to a chain of chemical reactions called lipid peroxidation (LPO). Malondialdehyde (MDA), an end product of lipid peroxidation represents level of lipid peroxidation.

Objectives- The objective of this study was to estimate the levels of seminal Malondialdehyde(MDA) in patients of infertility and to compare these values in normozoospermic and oligozoospermic patients and to correlate these values with the seminal parameters.

Methods- The study was carried out in the Department of Physiology, Govt. Medical College, Aurangabad. $n=60$ men (30 normozoospermic and 30 oligozoospermic) between the age group of 20-45 years referred for semen analysis in the Department of Physiology from the infertility clinic were studied. The semen analysis was preformed according to WHO criteria. Seminal MDA levels were measured by using thiobarbituric acid assay (TBARS). The MDA levels were compared between the normozoospermic and oligozoospermic patients and correlated with various sperm parameters.

Results- The MDA levels were increased in oligozoospermic patients and were inversely related to the sperm count and motility.
\end{abstract}

Conclusion-We conclude that the MDA levels may be correlated for the pathophysiology of male infertility and its assessment may guide in the medical treatment of infertility.

Key Words: Reactive Oxygen Species (ROS) Malondialdehyde (MDA), male infertility.

\section{INTRODUCTION}

Defective sperm function is the most prevalent cause of male infertility and a difficult condition to treat. Many environmental, physiological, and genetic factors have been implicated in the poor sperm function and infertility. Evaluation of the potential causes of sperm damage leading to abnormal sperm function and infertility is an important area of investigation. ${ }^{1}$

Free radical-induced oxidative damage to spermatozoa is one such condition which is recently gaining a considerable attention for its role in inducing poor sperm function and infertility. Controlled generation of reactive oxygen species (ROS) in spermatozoa is associated with normal physiological function. Uncontrolled and excessive production of ROS, however, seems to have a significant role as one of the major factors leading to an infertile status. ${ }^{2}$

Lipid peroxidation of sperm membrane is considered to be the key mechanism of this ROS-induced sperm damage leading to infertility. ${ }^{3}$ malondialdehyde (MDA), a by-product of lipid peroxidation represents level of lipid peroxidation. ${ }^{1}$

Studies carried out to evaluate primary infertility showed that seminal reactive oxygen species levels are significantly higher in infertile men as compared to normal men and the presence of excessive ROS in semen is positively correlated with low sperm concentration, poor motility and poor morphology. ${ }^{4,5,6}$

However the correlation or real role of MDA with seminal quality remains controversial. Considering the above mentioned facts, it seems that estimation of seminal MDA levels must be evaluated as a routine investigation in all cases of infertility in light of the increasing prevalence of the male infertility. With this background, the present study was planned to primarily evaluate the seminal MDA levels and then to compare these levels with the normozoospermic and oligoasthenozoospermic infertility patients.

\section{MATERIAL AND METHODS}

The present study was carried out in the Department of Physiology Govt.Medical College Aurangabad .The study protocol was approved by the Institutional Ethical Committee. Before enrollment in the study, informed written consent was obtained from each subject. 


\section{Source of patient:}

The patients referred for semen analysis attending the outpatient department (O.P.D.) of obstetrics and gynecology (OBGY), for infertility evaluation, were enrolled into the present study.

\section{Inclusion criteria:}

Clinically infertile patients with $\mathrm{H} / \mathrm{O}$ infertility persisting longer than one year in reproductive age group were included in the study.

\section{Exclusion criteria:}

Patients with the following conditions known to influence oxidative stress were excluded from the study.

1. Patients with $\mathrm{H} / \mathrm{O}$ smoking, alcohol consumption and tobacco chewing.

2. Patients suffering from diabetes mellitus, hypertension, tuberculosis, COPD, arthritis, infections and AIDS.

3. Patients taking drugs like vitamin $\mathrm{E}$, vitamin $\mathrm{C}$ or glutathione supplementation.

4. Patients with abnormalities like cryptorchidism (undescended testes), atrophic testes or varicocele.

5. Patients having genitourinary infection.

6. Semen samples having $>1$ million leukocytes $/ \mathrm{ml}$ of semen.

\section{Subjects:}

A total of sixty men (thirty normozoospermic and thirty oligoasthenozoospermic) between the age group 20-45 years were taken into study. The study was undertaken for duration of 6 months.

\section{Sample collection and semen analysis:}

Semen samples were collected by masturbation into a sterile, wide mouthed container, after at least 72 hours (34days) of sexual abstinence. Samples were allowed to liquefy at room temperature $\left(25^{\circ} \mathrm{c}\right)$ for at least 45 minutes. After liquefaction, samples were analyzed for volume, $\mathrm{pH}$, sperm count, motility and morphology (by using sperm meter), according to World Health Organization (WHO) guidelines. ${ }^{7}$

All the samples were divided in two groups:

1) Normozoospermic.

2) Oligoasthenozoospermic (count $<20 \times 10 \% \mathrm{ml}$, motility $<40 \%$ and $>30 \%$ normal morphology).

\section{Measurement of MDA:}

MDA levels were estimated by using thiobarbituric acid (TBARS) assay (by Rao et al method). ${ }^{8}$

\section{Statistical analysis:}

The MDA levels in both the groups were determined. Coefficients of correlation were calculated by using Spearman's correlation coefficient. The relationship between MDA levels and sperm motility and sperm concentration were compared. The statistical analysis for comparing two groups were performed on statistical software (Microsoft Excel 2003) and hypothesis were tested using 'unpaired t test' which were found to be significant against $5 \%$ level of significance (i.e. $\mathrm{p}<0.05$ ).

\section{OBSERVATIONS AND RESULTS}

The mean sperm concentrations of normozoospermic and Oligoasthenozoospermic males were $59.63 \pm 22.85$ and $9.4 \pm 6.11$ respectively which showed a significant difference between the two groups $(\mathrm{p}<0.001)$.

Means of percentage sperm motility $(64.93 \pm 14.33 \mathrm{v} / \mathrm{s} 21.9 \pm 9.4)$ also showed statistically significant difference $(\mathrm{p}<0.001)$ between the two groups. (Table 1$)$

The concentration of seminal MDA in normozoospermic males was $1.19 \pm 0.31$.On the other hand, it was found increased in Oligoasthenozoospermic males in whom it was $2.08 \pm 0.46$. So the concentrations of MDA in both groups were significantly different $(\mathrm{p}<0.001)$ as seen from table 2 and graph 1 . Individuals with oligoasthenozoospermia are related with the elevated MDA concentration. 
Table - 1

Sperm concentration, motility and MDA levels in normozoospermic and Oligoasthenozoospermic males

\begin{tabular}{|l|c|c|c|c|}
\hline \multicolumn{1}{|c|}{ Subject } & $\mathbf{n}$ & $\begin{array}{c}\text { Concentration } \\
(\mathbf{1 0} / \mathbf{m l}) \\
\text { Mean } \pm \text { SD }\end{array}$ & $\begin{array}{c}\text { Motility } \\
(\mathbf{\%}) \\
\text { Mean } \pm \text { SD }\end{array}$ & $\begin{array}{c}\text { MDA } \\
(\mathbf{n m o l} / \mathbf{m l}) \\
\text { Mean } \pm \text { SD }\end{array}$ \\
\hline Normozoospermic & 30 & $59.63 \pm 22.85$ & $64.93 \pm 14.33$ & $1.19 \pm 0.31$ \\
\hline Oligoasthenozoospermic & 30 & $9.4 \pm 6.11$ & $21.9 \pm 9.4$ & $2.08 \pm 0.46$ \\
\hline
\end{tabular}

$\mathrm{n}=$ number of subjects

SD - Standard Deviation

$$
\text { Table }-2
$$

Comparison of mean sperm count, motility and MDA between normozoospermic and oligozoospermic patients

\begin{tabular}{|c|c|c|c|c|}
\hline Parameters & $\begin{array}{l}\text { Normozoospermic } \\
\quad(\text { Mean } \pm \text { SD })\end{array}$ & $\begin{array}{l}\text { Oligozoospermic } \\
\text { (Mean } \pm \text { SD })\end{array}$ & 't $t$ ' value & 'p' value \\
\hline $\begin{array}{l}\text { Count } \\
\text { (millions/ml) }\end{array}$ & $59.63 \pm 22.85$ & $9.4 \pm 6.11$ & 11.63 & $<0.001$ \\
\hline $\begin{array}{l}\text { Motility } \\
(\%)\end{array}$ & $64.93 \pm 14.3$ & $21.9 \pm 9.46$ & 13.73 & $<0.001$ \\
\hline $\begin{array}{l}\text { MDA } \\
(\mathrm{nmol} / \mathrm{ml})\end{array}$ & $1.19 \pm 0.31$ & $2.08 \pm 0.46$ & 8.79 & $<0.001$ \\
\hline
\end{tabular}

SD - Standard Deviation

$\mathrm{p}<0.001$ : highly significant

Graph 1

Comparison of mean seminal MDA levels in normozoospermic and oligoasthenozoospermic patients

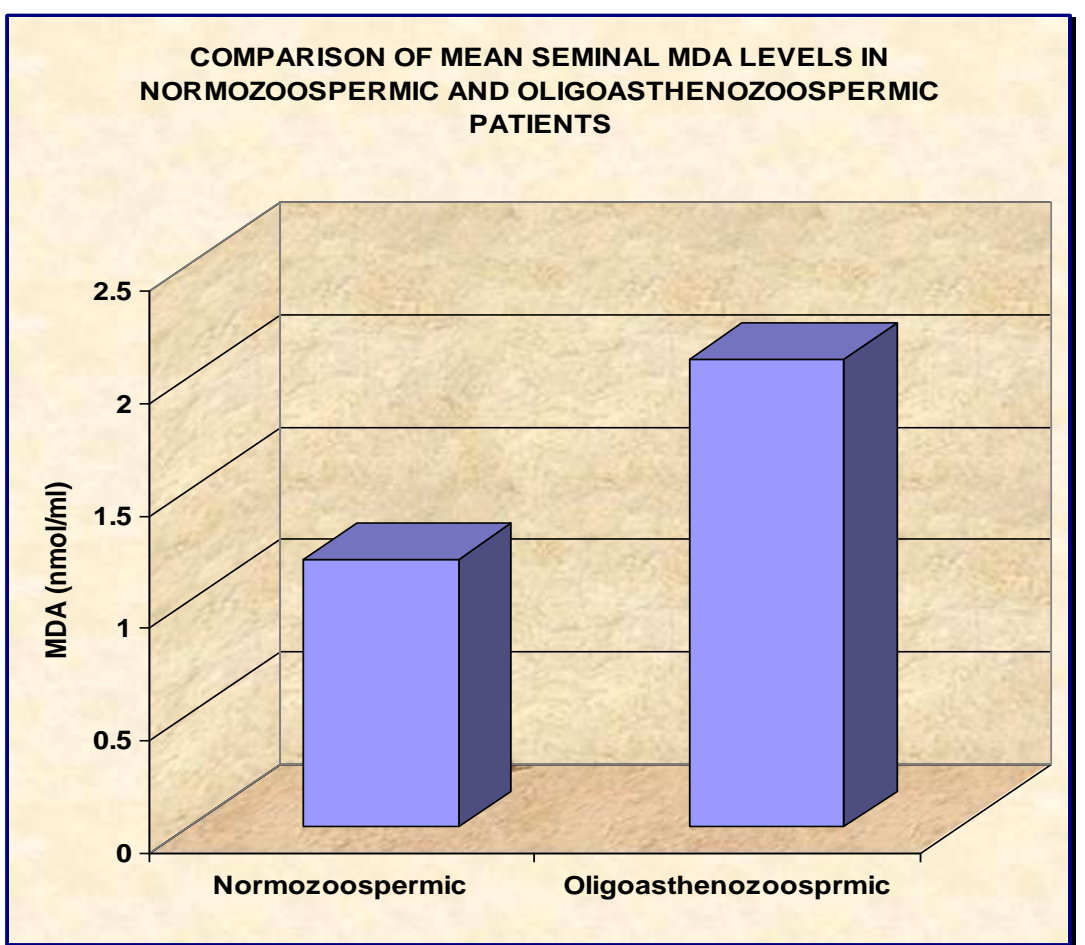

$* \mathrm{p}<0.001$ : Statistically highly significant 
Table - 3

Correlation between seminal MDA levels and sperm count

\begin{tabular}{|c|c|c|}
\hline $\begin{array}{c}\text { MDA }(\mathrm{nmol} / \mathrm{ml}) \\
(\text { Mean } \pm \text { SD) }\end{array}$ & $\begin{array}{c}\text { Count }(\text { millions } / \mathrm{ml}) \\
(\text { Mean } \pm \text { SD) }\end{array}$ & $\begin{array}{c}\text { Coefficient of } \\
\text { correlation }\end{array}$ \\
\hline $1.63 \pm 0.59$ & $34.51 \pm 30.27$ & $\mathrm{r}=-0.72$ \\
\hline
\end{tabular}

SD-Standard Deviation

Seminal MDA levels were also correlated with sperm concentration and percentage motility. Table 3 shows the correlation between MDA and sperm concentration. Seminal plasma MDA levels showed a negative correlation with sperm concentration $(r=-0.72)($ Graph 2$)$.

Graph 2

Correlation between seminal MDA levels and sperm count

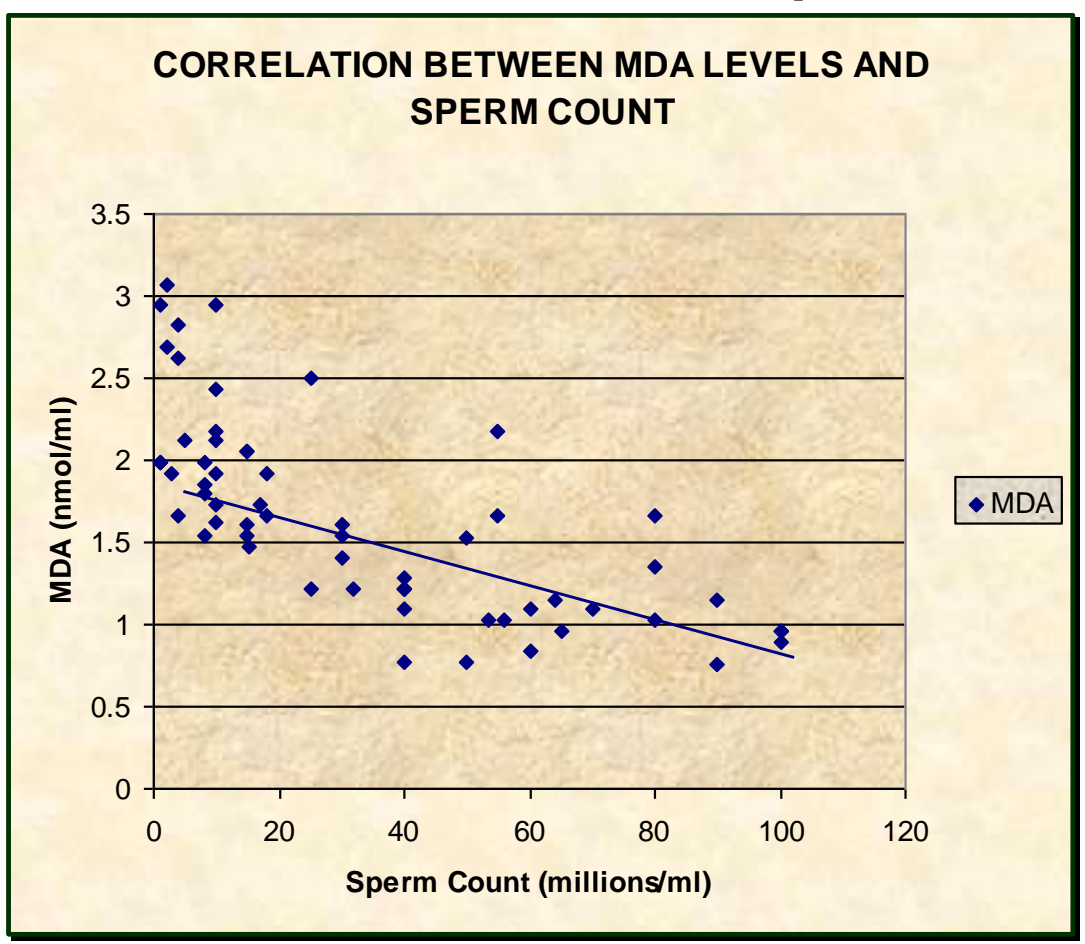

Table - 4

Correlation between seminal MDA levels and sperm motility

\begin{tabular}{|c|c|c|}
\hline MDA & Motility \% & Coefficient of \\
$($ Mean \pm SD) & (Mean \pm SD) & Correlation \\
\hline $1.63 \pm 0.59$ & $43.41 \pm 24.81$ & $\mathrm{r}=-0.929$ \\
\hline
\end{tabular}

SD-Standard Deviation

As shown in Table 4 and depicted in graph 3, significant negative correlation was found between seminal malondialdehyde levels and sperm motility $(r=-0.929)$. 
Graph 3

Correlation between seminal MDA levels and sperm motility

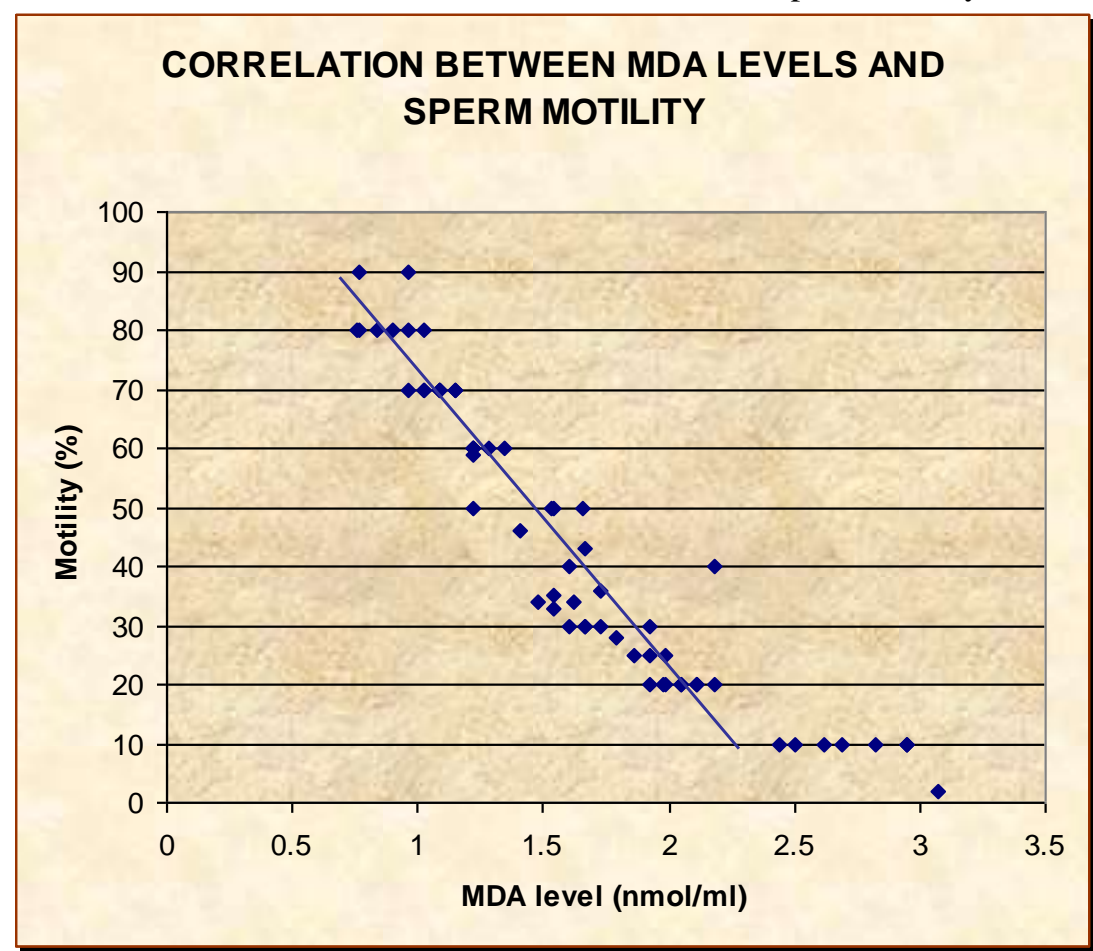

\section{DISCUSSION}

The present study was undertaken to estimate the levels of malondialdehyde (MDA) in infertility patients and to compare these values in normozoospermic and oligoasthenozoospermic patients.

The results obtained in the present study showed that the mean \pm SD concentration of seminal MDA in normozoospermic males was $1.19 \pm 0.31$ and in Oligoasthenozoospermic males the value was $2.08 \pm 0.46$. The intergroup comparison of the seminal MDA levels has shown that the difference of the mean concentrations of MDA in both the groups was statistically significant $(\mathrm{p}<0.001)$. The individuals with oligoasthenozoospermia thus are related with the elevated MDA levels.

Significant negative correlation was found between seminal malondialdehyde levels and sperm motility $(\mathrm{r}=-0.929)$. Also seminal plasma MDA levels showed a negative correlation with sperm concentration $(\mathrm{r}=-0.72)$

The above results of the present study are in accordance with the studies carried out by Fraczek $\mathrm{M}$ et al $(2001)^{77}$, Keskes-Ammar et al (2003) ${ }^{8}$. Nosratollah Zarghami et al (2005) ${ }^{9}$ andYao-Yuan Hseih et al (2006) ${ }^{10}$ who observed that seminal plasma level of MDA was higher in asthenozoospermic males than in normozoospermic males.

Analysis of the fatty acid content of human spermatozoa has revealed a high degree of polyunsaturation and this factor together with the capacity of these cells to generate reactive oxygen species (ROS) render them particularly susceptible to oxidative stress.

Unfortunately the spermatozoa are unable to repair the damage induced by oxidative stress because they lack the cytoplasmic enzyme systems that are required to accomplish this repair. ${ }^{11,12}$

ROS is produced by a variety of semen components including immotile or morphologically abnormal spermatozoa, leukocytes, and morphologically normal but functionally abnormal spermatozoa. Normally, a balance is maintained between the amount of ROS produced (pro-oxidants) and that scavenged by a cell (antioxidants). Cellular damage arises when this equilibrium is disturbed, especially when the cellular scavenging systems cannot eliminate the increase in ROS. ${ }^{13}$

ROS plays a central role for sperm physiology such as sperm maturation and capacitation. Abnormal ROS production is associated with defective sperm function. A fine balance between ROS production and recycling is essential for spermatogenesis. ${ }^{14}$

One of the byproducts of lipid peroxidation decomposition is malondialdehyde (MDA). This byproduct has been used in biochemical assays to monitor the degree of peroxidative damage in spermatozoa. The results of such an assay exhibit an excellent correlation with the degree to which sperm function is impaired in terms of 
motility and the capacity for sperm-oocyte fusion. While a reduction in any of the sperm parameters (count, motility, morphology) are commonly seen in men with oxidative stress, asthenozoospermia is probably the best surrogate marker of oxidative stress in a routine semen analysis. ${ }^{15}$

In general, the most significant effect of LPO in all cells is the perturbation of membrane (cellular and organellar) structure and function (transport processes, maintenance of ion and metabolite gradients, receptormediated signal transduction, etc.). Besides membrane effects, LPO can damage DNA and proteins, either through oxidation of DNA bases (primarily guanine via lipid peroxyl or alkoxyl radicals) or through covalent binding to MDA resulting in strand breaks and cross-linking. ${ }^{16}$ ROS can also induce oxidation of critical -SH groups in proteins and DNA, which will alter structure and function of spermatozoa with an increased susceptibility to attack by macrophages. ${ }^{17}$

High levels of ROS disrupt the inner and outer mitochondrial membrane resulting in release of cytochrome-C protein from the mitochondria that activates the caspases and induces apoptosis. ${ }^{18}$

Reduced motility due to lipid peroxidation may be due to a cascade of events that results in a decrease in axonemal protein, phosphorylation and sperm immobilization, both of which are associated with a reduction in membrane fluidity that is necessary for sperm-oocyte fusion. ${ }^{19}$ Another hypothesis is that hydrogen peroxide can diffuse across the membrane into the cell and inhibit the activity of some enzymes such as Glucose 6Phosphate dehydrogenase (G6PD). This enzyme controls the rate of glucose flux through the hexose monophosphate shunt, which in turn controls the intracellular availability of nicotinamide adenine dinucleotide phosphate (NADPH).This in turn is used as a source of electron by spermatozoa to fuel the generation of ROS by an enzyme system known as NADPH oxidase. ${ }^{20}$ Inhibition of G6PD leads to a decrease in the availability of NADPH and a concomitant accumulation of oxidized glutathione. This can reduce the antioxidant defenses of the spermatozoa and peroxidation of membrane phospholipids. ${ }^{21}$

Assessment of such oxidative stress status (OSS) may help in the medical treatment of this male factor infertility by suitable antioxidants. Basic and clinical research on the involvement of ROS and antioxidants in maintaining normal sperm function is very much warranted.

\section{BIBLIOGRAPHY}

[1] Sikka S C. Oxidative stress and role of antioxidants in normal and abnormal sperm function. Front Biosci 1996;1:78-86.

[2] Pasqualotto F F, Sharma R K, Nelson D R, Thomas A J, Agarwal A. Relationship between oxidative stress, semen characteristics, and clinical diagnosis in men undergoing infertility investigation. Fertil Steril 2000;73:459-464.

[3] Lenzi A, Cualosso F, Gandini L, Lombardo F, Dondero F. Placebo controlled, double-blind, cross-over trial of glutathione therapy, in male infertility. Hum Reprod 1993;9:2044-2050.

[4] Keskes-Ammar L, Feki-Chakroun N, Rebai T, Sahnoun Z, Ghozzi H, Hammami S, et al. Sperm oxidative stress and the effect of an oral vitamin E and selenium supplement on semen quality in infertile men. Arch Androl 2003;49(2):83-94.

[5] Zarghami N, Khosrowbeygi A. Seminal plasma levels of 15-F2 $\alpha$-isoprostane, malondialdehyde and total homocysteine in normozoospermic and asthenozoospermic males. Ind J Clin Biochem 2005;20(2):86-91.

[6] Hsieh Y, Chang C, Lin C. Seminal malondialdehyde concentration but not glutathione peroxidase activity is negatively correlated with seminal concentration and motility. Int J Biol Sci 2006;2(1):23-29.

[7] Nouri M, Gasemzadeh A, Farzadi L, Shehnazi V. Diagnostic value of non-enzymatic antioxidant capacity and rate of lipid peroxidation in plasma semen and spermatozoa of asthenozoospermic infertile males. Med J Tab Uni Med Sci 2008;29(4):17.

[8] Saraniya A, Koner B C, Doureradjou P, Selvaraj N, Shivagourou V. Altered malondialdehyde, protein carbonyl and sialic acid levels in seminal plasma of microscopically abnormal semen. Andrologia 2008;40:56-57.

[9] Yadav S B, Suryakar A N, Huddakar A D, Durgawale P P, Shukla P S. Antioxidant treatment a new therapeutic approach to reversible male infertility. Biomed Resear 2006;17(3):175-178.

[10] Suleiman S A, Ali M E, Zaki Z M S, El-Malik E M A, Nasr M A. Lipid peroxidation and human sperm motility: protective role of vitamin E. J Androl 1996;17(5):530-537.

[11] Reid c. Excited state in chemistry and biology. Butterworth London 1957.

[12] Jones R, Mann T, Sherins R. Peroxidative breakdown of phospholipids in human spermatozoa, spermicidal properties of fatty acid peroxides, and protective action of seminal plasma. Fertil Steril 1979;31:531-537.

[13] Dandekar S P, Nadkarni G D, Kulkarni V S, Punekar S. Lipid peroxidation and antioxidant enzymes in male infertility. J Postgrad Med 2002;48(3):186-190

[14] Alvarez J G, Touchstone J C, Blasco L, Storey B T. Spontaneous lipid peroxidation and production of hydrogen peroxide and Superoxide in human spermatozoa : superoxide dismutase as major enzyme protectant against oxygen toxicity. J Androl. 1987; 8:338-348.

[15] De Lamirande E, Gagnon C. Reactive oxygen species and human spermatozoa. II Depletion of adenosine triphosphate plays an important role in the inhibition of sperm motility. J Androl 1992; 13:379-386.

[16] Ernster L. Lipid peroxidation in biological membranes: mechanisms and implications. In: Active oxygen, lipid peroxides and antioxidants. Ed: Yagi K, CRC Press, Boca Raton 1993:1-38

[17] Aitken R J, West K M, Buckingham D W. Leukocyte infiltration into the human ejaculate and its association with semen quantity, oxidative stress, and sperm function. J Androl 1994;15:343-352

[18] Maneesh M, Jayalekshmi H. Role of reactive oxygen species and antioxidants on pathophysiology of male reproduction. Ind J Clin Biochem 2006;21(2):80-89

[19] De Lamirande E, Gagnon C. Impact of reactive oxygen species on spermatozoa: a balancing act between beneficial and detrimental effects. Hum Reprod 1995; 10:15-21.

[20] Sharma R K, Agarwal A. Reactive oxygen species and male infertility. Urology 1996;48:835-850.

[21] Grivaeu, J F, Dumont E, Renard B, Callegari J P and Lannou D L. Reactive oxygen species, lipid peroxidation and enzymatic defense systems in human spermatozoa. J Reprod Fertil 1995;103: 17-26. 